\title{
A NOTE ON DOEBLIN'S CENTRAL LIMIT THEOREM
}

\author{
DAVID G. KENDALL
}

Consider a Markov chain with a countable infinity of states $(1,2, \cdots)$ forming a single positive recurrent class, so that all the mean recurrence-times $m_{j j}$ are finite. Let $f(\cdot)$ be any real-valued function with the positive integers as domain, and if $X_{m}$ denotes the state of the system at the $m$ th epoch, let $S_{n} \equiv \sum_{m=0}^{n} f\left(X_{m}\right)$. Let $i$ be the initial state and $j$ any state, and let the state $j$ be occupied at the epochs $0 \leqq v_{1}<v_{2}<v_{3}<\cdots$ and at no others (this sequence will be nonterminating with probability one). We shall say that $\mu_{j}$ exists if $\left|f\left(X_{v_{1}+1}\right)+\cdots+f\left(X_{v_{2}}\right)\right|$ has a finite expectation, and we then write

$$
\mu_{j} \equiv \varepsilon\left(\sum_{m=v_{1}+1}^{v_{2}} f\left(X_{m}\right)\right) .
$$

Chung [2] has proved ${ }^{1}$ that if $\mu_{j}$ exists for one $j$, then it exists for all $j$. When the $\mu$ 's exist he has further shown that $S_{n} / n$ converges in probability (as $n \rightarrow \infty$ ) to the limit $M \equiv \mu_{j} / m_{j j}$ (which is therefore independent of $j$ ). Now write

$$
Z_{s} \equiv \sum_{m=v_{s}+1}^{v_{s+1}} f\left(X_{m}\right)-M\left(v_{s+1}-v_{s}\right) ;
$$

the $Z$ 's will be independent random variables having a common distribution and a finite first absolute moment and we shall have $\mathcal{E}(Z)$ $=0$, if the $\mu$ 's exist. Put $\sigma_{j}^{2} \equiv \operatorname{var}(Z) \leqq \infty$. Chung has shown ${ }^{2}$ that $\sigma_{1}, \sigma_{2}, \sigma_{3}, \cdots$ are either all finite or all infinite. Finally we have Doeblin's central limit theorem (Doeblin [4]; see also Chung [2]):

If the $\mu$ 's exist and the $\sigma$ 's are finite and non-zero AND IF THE RECURRENCE TIMES HAVE FINITE SECOND MOMENTS then $S_{n}$ is asymptotically normally distributed with mean $M n$ and with variance

$$
B n \equiv \sigma_{j}^{2} n / m_{j j}
$$

(thus $B$ is independent of $j$ ).

Received by the editors November 1, 1956.

${ }^{1}$ In his proof one must replace the first two sentences of p. 409 by "It is easy to show that if $X$ and $Y$ are independent then $\mathcal{E}(|X+Y|)<\infty$ implies $\mathcal{E}(|X|)<\infty$. Now $W_{1}, W_{2}$ and $W_{3}$ are independent and so, by $(21), \mathcal{E}\left(\left|W_{2}\right|\right)<\infty$."

${ }^{2}$ Note that his proof (second part of his Theorem 3) should be preceded by the proof of his Theorem 4 , because it requires the fact that $M$ does not depend upon $j$. If this precaution is not observed, an otherwise avoidable reference has to be made to the second moment of the recurrence-time. 
The purpose of this note is to show that:

In the statement and proof of Doeblin's central limit theorem the condition set out above in block capitals can be omitted.

We follow Chung by defining $l(n)$ to be the integer $l$ such that $v_{l} \leqq n<v_{l+1}$ (with $l \equiv 0$ if $n<v_{1}$ ), and we then write, as he does,

$$
S_{n}-M n=Y^{\prime}+Y^{\prime \prime}+\left(Z_{1}+Z_{2}+\cdots+Z_{l-1}\right)-M\left(n-v_{l}+v_{1}\right)
$$

(if $n \geqq v_{1}$ ), where

$$
Y^{\prime} \equiv \sum_{m=0}^{v_{1}} f\left(X_{m}\right), \quad Y^{\prime \prime} \equiv \sum_{m=v_{l}+1}^{n} f\left(X_{m}\right) .
$$

Chung's arguments then show at once that $Y^{\prime} / n^{1 / 2}, Y^{\prime \prime} / n^{1 / 2}$ and $\left(n-v_{l}+v_{1}\right) / n^{1 / 2}$ tend to zero in probability when $n \rightarrow \infty$. Thus (cf. Cramér [3, p. 254]) we have only to show that

$$
\left(Z_{1}+Z_{2}+\cdots+Z_{l-1}\right) / n^{1 / 2}
$$

has asymptotically a normal distribution with mean zero and variance $B$. Let $0<\epsilon<1$ and let $^{3}$

$$
\lambda(n) \equiv\left[\frac{n}{m_{j j}}(1+\epsilon)\right], \mu(n) \equiv\left[\frac{n}{m_{j j}}\right]+1, \nu(n) \equiv\left[\frac{n}{m_{j j}}(1-\epsilon)\right]+1,
$$

so that $1 \leqq \nu \leqq \mu \leqq \lambda$ if $n>m_{j j} / \epsilon$, and then

$$
0 \leqq(\lambda-\nu) / \mu \leqq 2 \epsilon .
$$

Now it is clear that $\mu(n) / n \rightarrow 1 / m_{j j}$ and that $\left(Z_{1}+Z_{2}+\cdots+Z_{\mu}\right) / \mu^{1 / 2}$ is asymptotically normally distributed about zero with variance $\sigma_{j}^{2}$; thus the sharpened form of Doeblin's central limit theorem will be proved if we can show that

$$
\left\{\left(Z_{1}+Z_{2}+\cdots+Z_{l-1}\right)-\left(Z_{1}+Z_{2}+\cdots+Z_{\mu}\right)\right\} / \mu^{1 / 2}
$$

tends to zero in probability as $n \rightarrow \infty$.

Now ( $\omega$ designating a sample-point in the probability-space)

$$
\begin{aligned}
\left\{\omega: \frac{n}{m_{j j}}(1-\epsilon)<l(n)-1<\frac{n}{m_{j j}}(1+\epsilon)\right\} & \\
& \subseteq\{\omega: \nu(n) \leqq l(n)-1 \leqq \lambda(n)\},
\end{aligned}
$$

and $\{l(n)-1\} / n \rightarrow 1 / m_{j j}$ with probability one (and so also in probability) by the strong law of large numbers for identical components because

${ }^{3}$ Here $[x]$ denotes the integral part of $x$. 


$$
\begin{aligned}
& \frac{v_{1}+\left(v_{2}-v_{1}\right)+\cdots+\left(v_{l}-v_{l-1}\right)}{l-1} \\
& \leqq \frac{n}{l-1}<\frac{v_{1}+\left(v_{2}-v_{1}\right)+\cdots+\left(v_{l+1}-v_{l}\right)}{l} \frac{l}{l-1}
\end{aligned}
$$

if $v_{2} \leqq n$. Thus there exists an integer $N(\boldsymbol{\epsilon})$ such that

$$
\operatorname{pr}\{\nu(n) \leqq l(n)-1 \leqq \lambda(n)\} \geqq 1-\epsilon \text { if } n \geqq N(\epsilon) .
$$

Now suppose that $n \geqq N(\epsilon)$ and that $n>m_{j j} / \epsilon$ and that $\delta>0$; then

$$
\begin{aligned}
\operatorname{pr}\left\{\mid\left(Z_{1}+\cdots+Z_{l-1}\right)-\right. & \left.\left.Z_{1}+\cdots+Z_{\mu}\right) \mid \geqq \delta \mu^{1 / 2}\right\} \\
& \leqq \epsilon+\operatorname{pr}\left\{2 \max _{1 \leqq t \leqq \lambda-\nu}\left|\sum_{s=1}^{t} Z_{\nu+8}\right| \geqq \delta \mu^{1 / 2}\right\} \\
& \leqq \epsilon+4\left(\sigma_{j} / \delta\right)^{2}(\lambda-\nu) / \mu \\
& \leqq\left(1+8 \sigma_{j}{ }^{2} / \delta^{2}\right) \epsilon,
\end{aligned}
$$

on using Kolmogorov's inequality (for this see, e.g., Halmos [5, p. 196]). The result is now established.

The delicate character of the above argument arises from the fact that we are concerned with the sum of a random number of random variables. Such questions have of course been treated in general; see, for example, Anscombe [1].

\section{REFERENCES}

1. F. J. Anscombe, Large-sample theory of sequential estimation, Proc. Cambridge Philos. Soc. vol. 48 (1952) pp. 600-607.

2. K. L. Chung, Contributions to the theory of Markov chains, II. Trans. Amer. Math. Soc. vol. 76 (1954) pp. 397-419.

3. H. Cramér, Mathematical methods of statistics, Princeton, 1946.

4. W. Doeblin, Sur deux problemes de M. Kolmogoroff concernant les chaines dênombrables, Bull. Soc. Math. France vol. 52 (1938) pp. 210-220.

5. P. R. Halmos, Measure theory, New York, 1950.

Magdalen College, Oxford University 